\title{
TEM Specimen Preparation for the Physical Sciences
}

\author{
Lucille A. Giannuzzi ${ }^{1}$, Scott D. Walck ${ }^{1}$, and Ron Anderson ${ }^{3}$ \\ ${ }^{1}$ Mechanical Materials and Aerospace Eng., University of Central Florida, Orlando, FL 32816-2450 \\ ${ }^{2}$ PPG Industries, Inc., Glass Technology Center, P. O. Box 11472, Pittsburgh, PA 15238-0472 \\ ${ }^{3}$ Microscopy Today, 21 Westview Drive, Poughkeepsie, NY 12603
}

There are many different methods of specimen preparation suitable for analysis via transmission electron microscopy (TEM) and related techniques (i.e., STEM, HRTEM, electron holography, etc.). Ideally, the specimen preparation technique that one chooses should be dictated by the specimen requirements. However, the technique chosen may often depend on the expertise and availability of equipment to the user and the speed in which a final specimen may be produced. In their careers, the authors have employed a variety of methods spanning a large range of materials systems and are comfortable addressing the questions in the "Ask the Experts" session on sample preparation for the physical sciences. In this paper, we present three different specimen preparation techniques that are commonly used for the physical sciences, each of which have advantages and disadvantages.

The small angle cleavage technique (SACT) is an easy and relatively inexpensive method for preparing often-superior cross section samples [1]. A major limitation is that it does require that the substrate material cleave or fracture. Although it lends itself quite naturally to semiconductors, other brittle and hard materials have been successfully prepared, including glass, sapphire, quartz, $\mathrm{SiC}$, and $\mathrm{TiO}_{2}$. Major advantages include rapid turnaround time, no ion milling artifacts, little or no contamination, minimal material, and a geometry that lends itself to all analytical TEM techniques. It is also an effective pre-thinning technique for site-specific FIB milling. FIGS. 1 and 2 illustrate the use of SACT where the lack of ion milling damage would otherwise hinder the analysis. FIG. 1 is an under-focused image of the partial oxidation of the surface of a plasma cleaned Si sample and FIG. 2 is a cross sectional view of a $3 \mathrm{keV} \mathrm{N}$-implanted and annealed single crystal rutile sample.

There are a number of advantages that come into play when tripod polishing is combined with FIB methods. The specimen is mounted on a tripod polisher and polished from both sides to yield a thin specimen about 6 to $8 \mu \mathrm{m}$ thick with the desired target centered. This process takes about 5 to 10 minutes per side with the final polish performed with a relatively coarse $3-\mu \mathrm{m}$ grit size diamond lapping film. FIG. 3 shows an as-tripod polished specimen, $8 \mu \mathrm{m}$ thick, with the eventual target location centered and covered with a protective metal layer. FIB processing follows to yield a specimen that is TEM transparent as seen in FIG. 4. The advantages here are that rapid tripod polishing produces a specimen that needs only up to a few micrometers removed from each side so that FIB times are short. Also, the material sputtered from such a specimen is ejected away from the specimen, as opposed to landing on nearby, thick-specimen neighboring regions where it may cause back-sputtering (e.g., redeposition) onto the FIB'ed surface. However, the big advantage is that the resulting specimen can be inserted into an ion mill and thinned from the back side to further thin the specimen if needed, but more importantly, to remove FIB artifacts and amorphous layers.

Focused ion beam in-situ lift-out (INLO) yields all the advantages of the combined tripod polishing/FIB methods with the added benefit that the original bulk sample need not be destroyed (e.g., in order to perform the tripod polishing) [2]. As an example, FIG. 5 shows an FIB image of a 
pre-ceramic polymer coating on stainless steel (SS) after the INLO step. This specimen was further thinned to electron transparency using the FIB. FIG. 6 is a zero loss energy filtered TEM image of the interfacial region showing the SS on the right, a thin (5-10 nm) oxygen rich layer, a $50 \mathrm{~nm} \mathrm{Cr}$ rich layer within an $\sim 1 \mu \mathrm{m}$ Si-C-O glassy phase [3].

\section{References}

[1] S. D. Walck and J. McCaffrey. Mat. Res. Soc. Proceedings, Vol. 480, (1997).

[2] B.W. Kempshall et al., Microsc. and Microanal. 8 (Suppl. 2), (2002), 390-391.

[3] LAG is funded by PNNL through DOE OIT. S.M. Schwarz FIB prepared the specimen in FIGS. 5,6. C. Henager of PNNL provided the sample in FIG. 5,6. Thanks also for FEI and Omniprobe for their support.

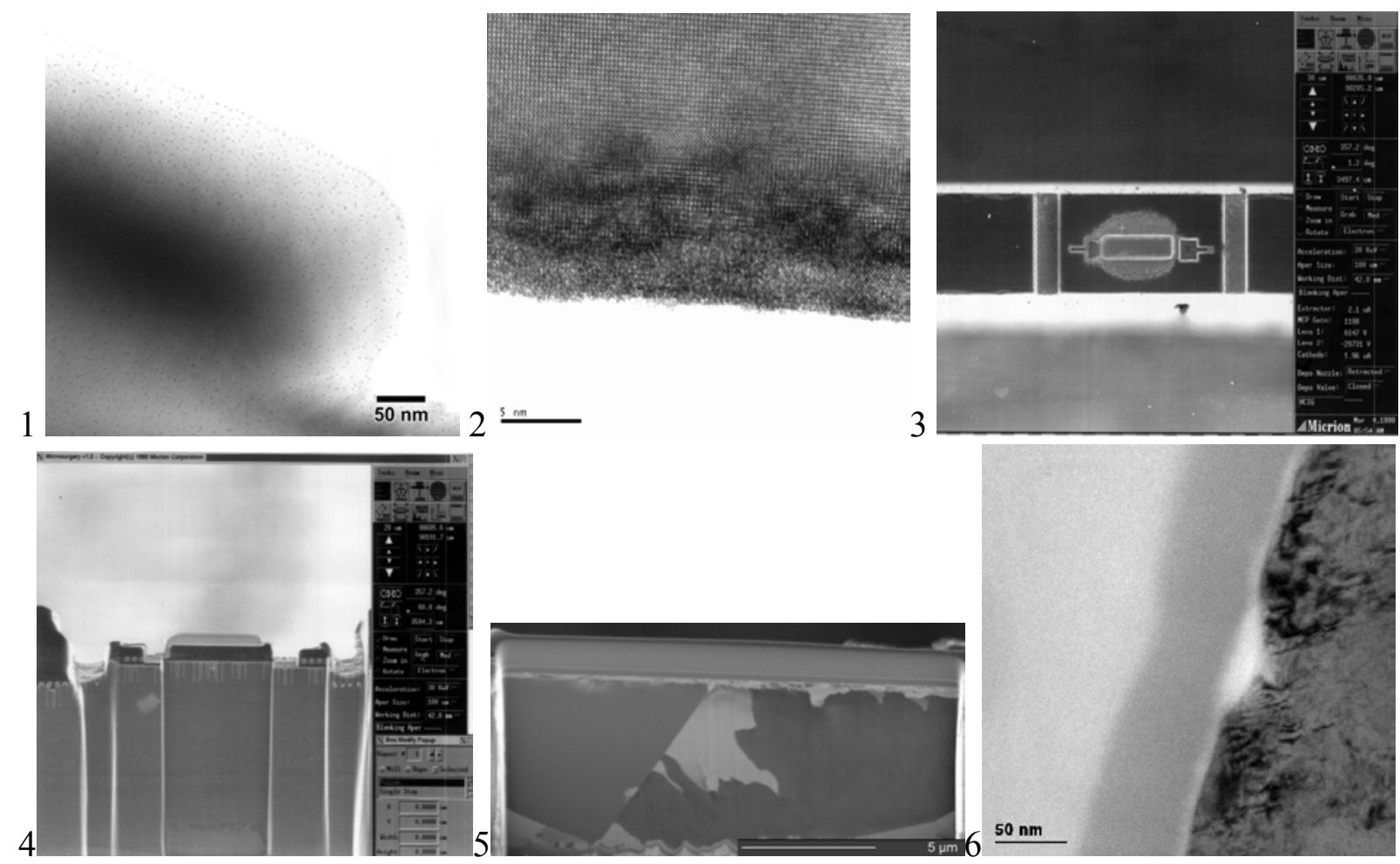

FIG. 1. An under-focused TEM image of the partial oxidation of the surface of a plasma cleaned Si sample specimen prepared by SACT.

FIG. 2. A XTEM image of a $3 \mathrm{keV} \mathrm{N}$-implanted and annealed single crystal rutile sample prepared by SACT.

FIG. 3. A tripod polished specimen ready for FIB thinning.

FIG. 4. The specimen in FIG. 3 after FIB milling to electron transparency.

FIG. 5. FIB image of a pre-ceramic polymer coating on stainless steel prepared by FIB INLO.

FIG. 6. Zero loss EFTEM image of the specimen in FIG. 5. 